\title{
Solución Matricial de Modelos para Cálculo de Equilibrio Líquido-Vapor
}

José F. Orejel-Pajarito y Raúl González-García

Universidad Autónoma de San Luis Potosí, Centro de Investigación y Estudios de Posgrado, Avda. Dr. Manuel Nava No. 6, 78210 San Luis Potosí, SLP-México

(fer_iqi@yahoo.com.mx, raulgg@uaslp.mx)

\begin{abstract}
Resumen
El objetivo de este artículo es demostrar la viabilidad de utilizar modelos termodinámicos de coeficientes de actividad (Wilson, NRTL, UNIQUAC) programados con matrices, en lugar de estar programados con ciclos. Se determina la relación de equilibrio líquido-vapor de las mezclas MetanolEtanol-Benceno y Acetona-Cloroformo-Metanol representados en mapas de curvas de residuo y en mapas de líneas de destilación. Para obtener resultados más confiables y conclusiones objetivas, el estudio fue apoyado con el uso del diseño estadístico de experimentos. La programación con matrices mostró ser mucho más rápida que el modelo cíclico convencional, independiente del tipo de modelo de coeficiente de actividad usado.
\end{abstract}

Palabras clave: coeficiente de actividad, modelos termodinámicos, equilibrio liquido-vapor, matrices

\section{Matrix Solution of Models to Calculate Liquid-Vapor Equilibrium}

\begin{abstract}
The objective of this paper is to show the viability of using thermodynamics models of activity coefficients (Wilson, NRTL, UNIQUAC) programmed with matrixes, instead of being programmed with cycles. The vapor-liquid equilibrium relation of mixtures Methanol-Ethanol-Benzene and Acetone-Chloroform-Methanol, represented with residue curve maps and distillation lines maps, was calculated. To obtain better solutions and objectives conclusions, the study was been supported with statistical design of experiments. The matrix programming showed to be faster than the conventional cyclic model, independent of the activity coefficient model used.
\end{abstract}

Keywords: activity coefficient matrix, thermodynamics models, vapor-liquid equilibrium, matrixes 


\section{INTRODUCCIÓN}

La experimentación tiene como fin, en muchos casos, desarrollar un proceso robusto, en el que las variables externas lo afecten lo menos posible. Una manera de lograr conclusiones válidas y a la vez objetivas de los experimentos, es realizando un Diseño Estadístico de Experimentos, el cual consiste en planear la manera de hacer el experimento, para que así sea posible recabar la información adecuada y concisa; más específicamente, el diseño experimental factorial, es una estrategia en la que las variables de entrada (factores) que intervienen en la experimentación, se hacen variar en conjunto, permitiendo así obtener resultados y conclusiones fehacientes (Montgomery, 2004). El diseño de experimentos se debe principalmente al trabajo de Sir Ronald A. Fisher entre 1920 y 1930, y fue él quien desarrolló las ideas para obtener los tres principios básicos del diseño experimental, los cuales son: La Aleatorización, La Realización de Replicas, y La Formación de Bloques.

Las matrices aparecen por primera vez hacia el año 1850, introducidas por J. J. Silvestre. El desarrollo inicial de la teoría se debe al matemático W. R. Hamilton en 1853. En 1858, A. Cayley introduce la notación matricial como una forma abreviada de escribir un sistema de $m$ ecuaciones lineales con $n$ incógnitas. Las matrices se utilizan en el cálculo numérico, en la resolución de sistemas de ecuaciones lineales, de las ecuaciones diferenciales y de las derivadas parciales; presentados en la literatura (Kreider et al., 1971).Los modelos que representan la relación que existe entre la fase líquida y vapor en el ELV, han sido ampliamente utilizados en la determinación analítica de los procesos químicos. La representación de esta relación es:

$\mathrm{y}_{\mathrm{i}}=\mathrm{K}_{\mathrm{i}} \mathrm{x}_{\mathrm{i}}$

donde $y_{i}, x_{i}$ son fracciones molares vapor-liquido del componente $i$ respectivamente, $\mathrm{K}_{\mathrm{i}}$ es una medida de la tendencia del componente a evaporarse. $K_{i}$ se le conoce también como la relación de equilibrio y esta se puede representar de varias maneras. Una de las formas más comunes de representarla es utilizando la Ley de Raoult modificada (Widagdo y Seider, 1996):

$K_{i}=\frac{\gamma_{i} P_{i}^{\text {vap }}}{P}$

donde $\gamma_{i}$ es el coeficiente de actividad, $P$ es la presión del sistema, y $P_{i}^{\text {vap }}$ es la presión de vapor del componente $i$. Para la determinación del coeficiente de actividad $\left(\gamma_{i}\right)$, es preciso utilizar modelos tales como Wilson, NRTL, UNIQUAC, etc.

En este trabajo se implementó el diseño estadístico experimental factorial, para demostrar fehacientemente la viabilidad de utilizar modelos matriciales en lugar de modelos cíclicos; siendo el tiempo de cómputo la variable de respuesta que se medirá para dicha demostración. El diseño y análisis experimental se basó en un arreglo de 4 factores (tipo de programación, tipo de modelo de coeficiente de actividad, tipo de diagrama de equilibrio, tipo de mezcla), y cada factor con diferentes niveles (Matricial o ciclos; NRTL, Wilson o UNIQUAC; Líneas de Destilación o Curvas de Residuo; MetanolEtanol-Benceno o Acetona-Cloroformo-metanol) respectivamente.

\section{METODOLOGÍA}

Debido a la amplia aplicación que tiene el diseño de experimentos en diversas disciplinas, es posible aplicarlo en procesos de experimentación computacional para así comparar, el tipo de programación, los diferentes modelos de coeficientes de actividad, el tipo de mezclas, etc. Para ello es imperativo tener una idea clara de lo que se pretende estudiar, cómo deben establecerse los datos, y una compresión cualitativa de la forma en que va a analizarse la información. Es por ello que la metodología para diseñar los experimentos es la siguiente: [1] Identificación y enumeración del problema, [2] Elección de los factores, los niveles y los rangos, [3] Selección de la variable de respuesta, [4] Elección del diseño experimental, [5] Realización del experimento, [6] Análisis estadístico de los datos, [7] Conclusiones y recomendaciones. 
La Tabla 1 muestra un resumen del diseño que se obtiene una vez que se introducen los datos.

Tabla1: Representación del resumen de diseño

\begin{tabular}{|c|c|c|c|c|c|c|c|c|c|}
\hline \multirow{2}{*}{\multicolumn{4}{|c|}{ Tipo de Estudio: Factc }} & \multicolumn{2}{|c|}{ Experimentos: 24} & \multirow{2}{*}{\multicolumn{4}{|c|}{ Total de Experimentos: 72}} \\
\hline & & & & \multicolumn{2}{|c|}{ Replicas: 3} & & & & \\
\hline \multicolumn{2}{|c|}{ Respuesta } & Nombre & Unidad & Obs. & Mínimo & Máximo & \multicolumn{2}{|c|}{ Transformación } & Modelo \\
\hline Y1 & & Tiempo & Seg. & 72 & 0.7611 & 266.386 & \multicolumn{2}{|l|}{$\log _{10}$} & 3FI \\
\hline Factor & \multicolumn{2}{|c|}{ Nombre } & Tipo & $\begin{array}{l}\text { Nivel } \\
\text { bajo }\end{array}$ & $\begin{array}{l}\text { Nivel } \\
\text { medio }\end{array}$ & Nivel alto & & & \\
\hline$A$ & \multicolumn{2}{|c|}{ Prog. } & Categórico & Matriz & & Ciclo & Niveles: & \multicolumn{2}{|l|}{2} \\
\hline $\mathrm{B}$ & \multicolumn{2}{|c|}{ EcEdo } & Categórico & NRTL & Wilson & UNIQUAC & Niveles: & \multicolumn{2}{|l|}{3} \\
\hline $\mathrm{C}$ & \multicolumn{2}{|c|}{ DiagEq } & Categórico & LinDes & & CurRes & Niveles: & \multicolumn{2}{|l|}{2} \\
\hline D & \multicolumn{2}{|c|}{ Mezcla } & Categórico & MEB & & $\mathrm{ACM}$ & Niveles: & \multicolumn{2}{|l|}{2} \\
\hline
\end{tabular}

Modelos de coeficientes de actividad

La representación típica de un modelo que contiene un número de $n$ componentes es por medio de sumatorias, por ejemplo $\sum_{i=1}^{n} y_{i}=\sum_{i=1}^{n} K_{i} x_{i}=1$. Una forma análoga de representarla mediante un software es utilizando ciclos, tales como los ciclos "for", "while", etc. Otra manera de representarlo es por medio de algebra matricial: $\stackrel{=}{y}=\stackrel{=}{K} \cdot \overrightarrow{=}$, donde $\stackrel{\bar{K}}{\mathrm{~K}}$ es la matriz $m \times n$ que involucra las relaciones de equilibrio, y ${ }^{=}$es una matriz transpuesta $n \times m$ de las fracciones líquidas.

De manera similar es posible representar los cálculos para obtener los coeficientes de actividad para distintos modelos (Gmehling y Onken, 1988; Harding et al., 1997; Doherty y Malone, 2001).

\section{Modelo NRTL}

El modelo de NRTL (Non-Random-Two-Liquid) fue derivado por Renon y Prausnitz (1968) para la determinación de coeficientes de actividad para fases líquidas. En seguida se presenta su representación matemática:

$\ln \gamma_{i}=\frac{\sum_{j=1}^{n} \tau_{j i} G_{j i} x_{j}}{\sum_{j=1}^{n} G_{j i} x_{j}}+\sum_{j=1}^{n} \frac{G_{i j} x_{j}}{\sum_{k=1}^{n} G_{k j} x_{k}}\left(\tau_{i j}-\frac{\sum_{k=1}^{n} \tau_{k j} G_{k j} x_{k}}{\sum_{k=1}^{n} G_{k j} x_{k}}\right)$

$G_{i j}=\exp \left(-\alpha_{i j} \tau_{i j}\right)$

$\tau_{i j}=\frac{A_{i j}}{R T}$

donde $A_{i j}$ representa los parámetros de interacción binaria de los componentes, $\alpha_{i j}$ parámetro relacionado con la distribución al azar, $G_{i j}$ y $\tau_{i j}$ son parámetros de interacción, $\mathrm{R}$ la constante de los gases y $\mathrm{T}$ la temperatura. Utilizando las ecuaciones (3), (4) y (5), es posible representarlas en software MATLAB, como se muestra en la Tabla 2.

\section{Modelo Wilson}

Este modelo fue desarrollado por Wilson (Wilson, 1964) para representar la energía libre de Gibbs en exceso en fase líquida. La representación de la ecuación del coeficiente de actividad se muestra en las ecuaciones (6) y (7). 
Tabla 2:. Programación con análisis matricial y con ciclos para el modelo NRTL

\begin{tabular}{|c|c|c|}
\hline function $\mathrm{GAMA}=\mathrm{NRTL}(\mathrm{x}, \mathrm{T}, \mathrm{A}, \alpha)$ & \multicolumn{2}{|c|}{ function GAMA_for $=\mathrm{NRTL}(\mathrm{x}, \mathrm{T}, \mathrm{A}, \alpha)$} \\
\hline$\tau=\mathrm{A} /(\mathrm{RT})$ & $\tau=\mathrm{A} /(\mathrm{RT})$ & \\
\hline$G=\exp \left(-\alpha \cdot{ }^{*} \tau\right)$ & $G=\exp \left(-\alpha \cdot{ }^{*} \tau\right)$ & \\
\hline$o=$ ones $(\operatorname{size}(x))$ & for $i=1$ : length $(x)$ & $q(k, j)=\tau(k, j) * G(k, j) * x(:, k)$ \\
\hline$x O=x^{\top} * 0$ & for $\mathrm{J}=1$ : length $(\mathrm{x})$ & end \\
\hline $\mathrm{G} \times 0=G_{i j} \cdot{ }^{*} \times 0$ & $\begin{array}{l}a(,, i)-G(, 1) \cdot x(\cdot, j) \\
h(i, i)=\tau(i, i) * a(i, i)\end{array}$ & $F(\cdot i)=\operatorname{sum}(h(\cdot i)) / \operatorname{sum}(a(\cdot i))$. \\
\hline$D=\left(\operatorname{sum}\left(\tau \cdot{ }^{*} \mathrm{G} \times 0\right) \cdot / \operatorname{sum}(\mathrm{G} \times 0)\right)$ & $c(i, j)=G(i, j) * x(:, j) ;$ & $\mathrm{D}(\mathrm{i},:)=\mathrm{c}(\mathrm{i},:) \cdot / \operatorname{sum}(\mathrm{d})$ \\
\hline$\gamma=\exp \left(\mathrm{D}+\operatorname{sum}\left(\left(\left(G{ }^{*}{ }^{*} \mathrm{O}^{\top}\right) \cdot / \cdots\right.\right.\right.$ & for $k=1$ : length $(x)$ & $F(:, i)=\operatorname{tao}(:, i)-$ (ones... \\
\hline$\left.\left.\left.\left(0^{\top} * \operatorname{sum}(\mathrm{G} \times 0)\right) \cdot{ }^{*} \tau-\mathrm{O}^{\top} * D\right)^{\top}\right)\right)$ & $d(k, j)=G(k, j) * x(:, k)$ & $(\operatorname{size}(x)))^{\star} E(:, i)$ \\
\hline & & $\gamma=\exp \left(E+\operatorname{sum}\left(\left(D .^{*} F\right)^{\prime}\right)\right)$ \\
\hline
\end{tabular}

$$
\begin{aligned}
& \ln \gamma_{i}=1-\ln \left(\sum_{j=1}^{n} x_{j} \Lambda_{i j}\right)-\sum_{j=1}^{n}\left(\frac{x_{j} \Lambda_{j i}}{\sum_{k=1}^{n} x_{k} \Lambda_{j k}}\right) \\
& \Lambda_{i j}=\frac{V_{j}}{V_{i}} \exp \left(-\frac{A_{i j}}{R T}\right)
\end{aligned}
$$

donde $V_{j}$ es el volumen molar del componente $j, \Lambda_{i j}$ es un parámetro ajustable adimensional. Aplicando una analogía semejante a la utilizada en la programación del método NRTL, es posible resolver el modelo de Wilson de forma matricial y ciclos, tal como lo muestra la Tabla 3.

Tabla 3: Programación con análisis matricial y con ciclos para el modelo Wilson

function GAMA = Wilson $(x, T, A, V)$
$v=V{ }^{*}(\operatorname{ones}(\text { size }(V)))^{\top}$
Vo $=v^{\top} . / v ;$
$B=V_{0}{ }^{*} \exp (-A / T)$
$C=x{ }^{*} B^{\top} ;$
$\gamma=\exp \left(1-\log (C)-(x . / C){ }^{*} B\right)$

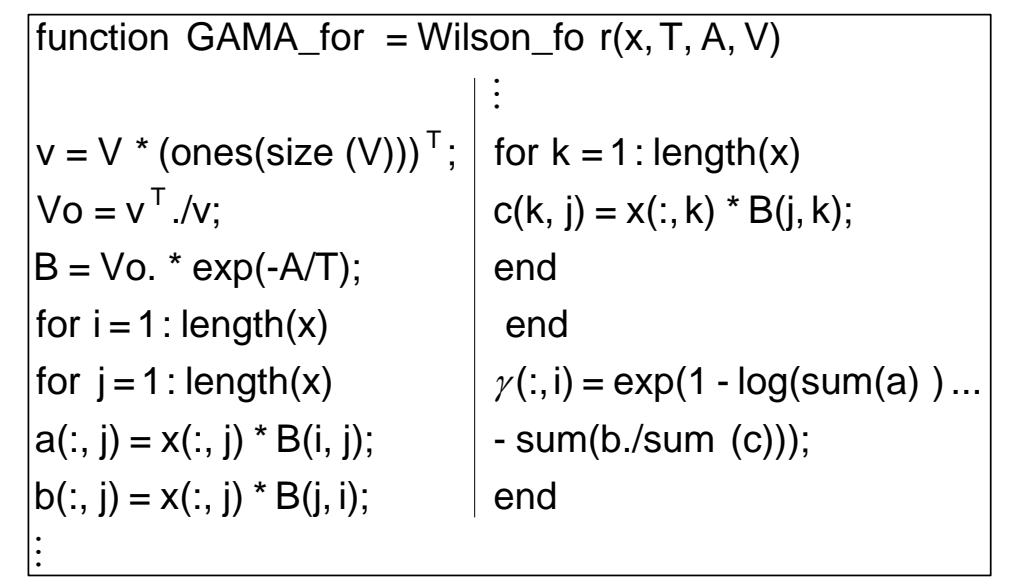

\section{Modelo UNIQUAC}

Fue desarrollado por Abrams y Prausnitz (1975) y el modelo es utilizado para calcular la energía libre de Gibbs en exceso y los coeficientes de actividad, se emplea para sistemas poco ideales. La ecuación para calcular el coeficiente de actividad se muestra a continuación:

$$
\begin{aligned}
& \ln \gamma_{i}=\ln \gamma_{i}{ }^{C}+\ln \gamma_{i}{ }^{R} \\
& \ln \gamma_{i}{ }^{C}=\ln \frac{\phi_{i}}{x_{i}}+\frac{z}{2} q_{i} \ln \frac{\theta_{i}}{\phi_{i}}+l_{i}-\frac{\phi_{i}}{x_{i}} \sum_{j=1}^{n} l_{j} x_{j}
\end{aligned}
$$


$\ln \gamma_{i}^{R}=q_{i}^{p}\left(\ln \left(\sum_{j=1}^{n} \tau_{j i} \theta_{j}^{p}\right)-\sum_{j}^{n} \frac{\tau_{i j} \theta_{j}^{p}}{\sum_{k=1}^{n} \tau_{k j} \theta_{k}^{p}}\right)$

$\theta_{i}=\frac{q_{i} x_{i}}{\sum_{j=1}^{n} q_{j} x_{j}} ; \quad \theta_{i}{ }^{p}=\frac{q_{i}{ }^{p} x_{i}}{\sum_{j=1}^{n} q_{j}{ }^{p} x_{j}}$

$\phi_{i}=\frac{r_{i} x_{i}}{\sum_{j}^{n} r_{j} x_{j}}$

$I_{i}=\frac{z}{2}\left(r_{i}-q_{i}\right)-\left(r_{i}-1\right)$

$\tau_{i j}=\exp \left(-\frac{A_{i j}}{T}\right)$

donde $z$ es el índice de coordinación con un valor normalmente de $10, r, q, q^{P}$ son constantes relativas a la estructura molecular de los componentes puros. En la Tabla 4 se muestra la representación del modelo matricial y con ciclos.

Tabla 4: Programación con análisis matricial y con ciclos para el modelo UNIQUAC
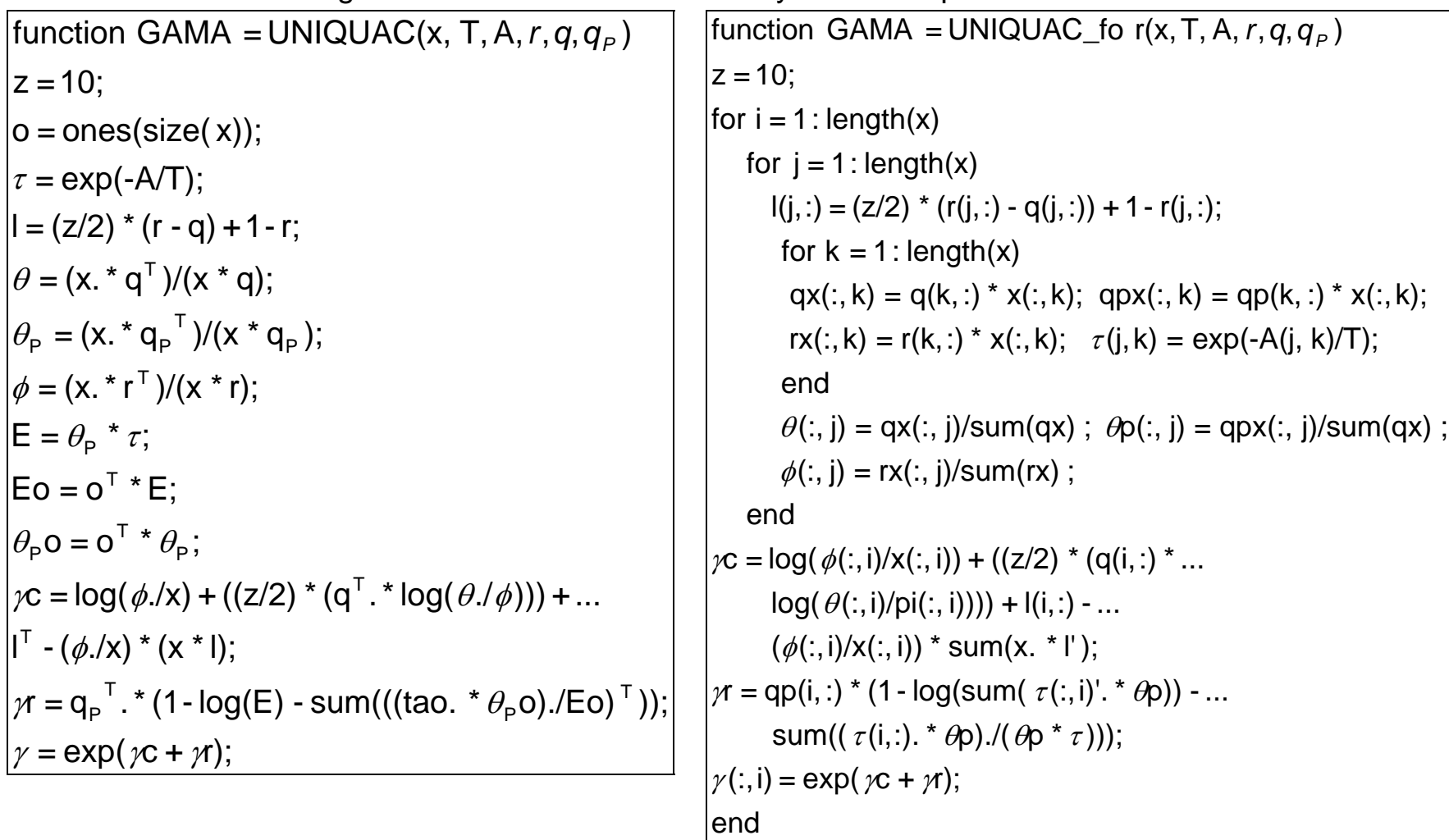

Donde $(\mathrm{x}, \gamma, \mathrm{r}, \mathrm{q}, \mathrm{V})$ son vectores; $(\mathrm{A}, \alpha, \tau) \in \mathrm{R}^{\mathrm{nxn}} ;\left(^{*}\right),(. /)$, (*), (sum), son operaciones en MATLAB de producto y división de elementos de la matriz (producto punto), multiplicación de matrices y suma de columnas respectivamente.

Es preciso señalar que la programación presentada es aplicable para $n$ componentes. Además de que la programación puede ser más compacta o extensa dependiendo del programador. 


\section{RESULTADOS Y DISCUSIÓN}

Se presenta una serie de resultados obtenidos comparando los modelos de NRTL, Wilson y UNIQUAC resueltos con programación por bloques (matricial), y modelos resueltos con programación convencional (ciclos "for") para dos tipos de mezclas: Acetona-Cloroformo-Metanol, y Metanol-Etanol-Benceno. Las comparaciones se hicieron calculando las Curvas de residuo y Líneas de destilación

Los códigos de programación con matrices permiten obtener los coeficientes de actividad de las mezclas con una sola corrida del algoritmo. Pero al realizar los cálculos de los coeficientes de actividad con los códigos de programación con ciclos, es necesario realizar 27 corridas para llegar al mismo resultado; las cuales pueden incrementarse al aumentar el número de componentes.

Aplicando la metodología para diseñar experimentos presentada anteriormente, se presenta a continuación el análisis de varianza de las variables de diseño. En base a la Tabla 1, la Tabla 5 muestra la respuesta del programa, Debido a que un valor de "Prob > F" menor que 0.05 indica los términos del modelo que son significantes y mayores de 0.1 indica términos no significantes. En este caso $A, B, C$, $D, A B, A C, B C, B D, C D, A B C, A B D, A C D, B C D$ son significantes.

Tabla 5: Representación de la ANOVA

\begin{tabular}{|c|c|c|c|c|c|c|c|c|}
\hline \multicolumn{2}{|l|}{ Respuesta: } & \multicolumn{4}{|c|}{ Transformación: Log10 } & \multicolumn{2}{|c|}{ Lambda: 0.07} & Constante: 0 \\
\hline \multicolumn{9}{|c|}{ ANOVA para el modelo factorial seleccionado } \\
\hline \multicolumn{9}{|c|}{ Tabla de Análisis de Varianza [Suma parcial de cuadrados] } \\
\hline \multirow[b]{2}{*}{ Modelo } & $\begin{array}{c}\text { Suma de } \\
\text { cuadrados }\end{array}$ & DF & \multicolumn{2}{|l|}{$\begin{array}{c}\text { Media } \\
\text { Cuadrada }\end{array}$} & \multicolumn{2}{|c|}{ Valor de F } & Prob > F & \multirow[b]{2}{*}{ Significante. } \\
\hline & 49.3140804 & 21 & \multicolumn{2}{|l|}{2.34828954} & \multicolumn{2}{|c|}{67680.7835} & $<0.0001$ & \\
\hline A & 1.71965573 & 1 & \multicolumn{2}{|l|}{1.71965573} & \multicolumn{2}{|c|}{49562.7328} & $<0.0001$ & \\
\hline B & 2.51194657 & 2 & \multicolumn{2}{|l|}{1.25597329} & \multicolumn{2}{|c|}{36198.7968} & $<0.0001$ & \\
\hline C & 43.1239558 & 1 & \multicolumn{2}{|l|}{43.1239558} & \multicolumn{2}{|c|}{1242888.95} & $<0.0001$ & \\
\hline $\mathrm{D}$ & 0.03317248 & 1 & \multicolumn{2}{|l|}{0.03317248} & 956.07 & 4318 & $<0.0001$ & \\
\hline $\mathrm{AB}$ & 0.76981276 & 2 & 0.38490638 & & 11093. & 5066 & $<0.0001$ & \\
\hline$A C$ & 0.01089797 & 1 & 0.01089797 & & 314.09 & 3681 & $<0.0001$ & \\
\hline$A D$ & 1.8661E-07 & 1 & 1.8661E-07 & & 0.0053 & 7821 & 0.9418 & \\
\hline $\mathrm{BC}$ & 0.70055542 & 2 & 0.35027771 & & 10095. & 4629 & $<0.0001$ & \\
\hline $\mathrm{BD}$ & 0.0732556 & 2 & 0.0366278 & & 1055.6 & 6116 & $<0.0001$ & \\
\hline$C D$ & 0.27945673 & 1 & 0.27945673 & & 8054.3 & 0931 & $<0.0001$ & \\
\hline$A B C$ & 0.00818017 & 2 & 0.00409008 & & 117.88 & 1554 & $<0.0001$ & \\
\hline$A B D$ & 0.00022355 & 2 & 0.00011177 & & 3.2214 & 6773 & 0.0483 & \\
\hline ACD & 0.00010733 & 1 & 0.00010733 & & 3.0934 & 6446 & 0.0847 & \\
\hline $\mathrm{BCD}$ & 0.0828601 & 2 & 0.04143005 & & 1194.0 & 6842 & $<0.0001$ & \\
\hline Residual & 0.00173483 & 50 & 3.4697E-05 & & & & & \\
\hline $\begin{array}{l}\text { Falta de } \\
\text { ajuste }\end{array}$ & 7.9796E-05 & 2 & $3.9898 \mathrm{E}-$ & & 1.15 & 5713954 & 0.3230 & $\begin{array}{c}\text { No } \\
\text { significante }\end{array}$ \\
\hline Error & 0.00165503 & 48 & $3.448 \mathrm{E}$ & 05 & & & & \\
\hline Cor. Total & 49.3158152 & 71 & & & & & & \\
\hline Dev. Std. & 0.00589038 & R-cu & drada & 0.9 & 9996482 & & & \\
\hline Media & 0.94837465 & R-cu & idrada Aju. & 0.9 & 9998303 & & & \\
\hline C.V. & 0.62110238 & R-cu & drada Pred. & & 9997522 & & & \\
\hline PRESS & 0.00359734 & Prec & sión Adeq. & & 9.909033 & & & \\
\hline
\end{tabular}

Para lograr un mejor entendimiento de la tabla anterior, se presenta en seguida la Tabla 6, la cual muestra los resultados de los 72 experimentos realizados.

La mejor manera de detectar ideonidad de los supuestos del ANOVA (análisis de la varianza), es utilizando una grafica de los residuales contra el número de corridas (Figura 1). Por ejemplo, indica 
un aleatorización adecuada del experimento, la cual es un paso importante para conseguir la independencia de las corridas.

Tabla 6: Tabla de tiempos del total de los experimentos

\begin{tabular}{|c|c|c|c|c|c|c|c|c|c|}
\hline & Mezcla & Prog. & \multicolumn{6}{|c|}{ DiagEq } \\
\hline & & \multicolumn{4}{|c|}{$\mathrm{ACM}$} & \multicolumn{4}{|c|}{ MEB } \\
\hline & & \multicolumn{2}{|c|}{ Ciclo } & \multicolumn{2}{|c|}{ Matriz } & \multicolumn{2}{|c|}{ Ciclo } & \multicolumn{2}{|c|}{ Matriz } \\
\hline Replica & EcEdo & CurResi & LinDest & CurResi & LinDest & CurResi & LinDest & CurResi & LinDest \\
\hline \multirow[t]{3}{*}{ R1 } & NRTL & 28.5811 & 1.7325 & 24.6254 & 1.4120 & 35.3709 & 0.9213 & 30.0132 & 0.7611 \\
\hline & UNIQUAC & 217.8833 & 3.9657 & 65.9348 & 0.9213 & 266.3860 & 4.1560 & 77.4113 & 0.9514 \\
\hline & Wilson & 46.4167 & 2.7640 & 25.6669 & 1.4020 & 55.0692 & 1.5522 & 30.9345 & 0.7911 \\
\hline \multirow[t]{3}{*}{$\mathrm{R} 2$} & NRTL & 29.7127 & 1.7725 & 24.4752 & 1.4621 & 34.9102 & 0.9113 & 29.5124 & 0.7611 \\
\hline & UNIQUAC & 217.6430 & 3.9957 & 66.9162 & 0.9414 & 266.2929 & 4.1960 & 77.7818 & 0.9514 \\
\hline & Wilson & 46.7272 & 2.6738 & 25.5668 & 1.4120 & 57.2724 & 1.5222 & 31.5654 & 0.8412 \\
\hline \multirow[t]{3}{*}{ R3 } & $\begin{array}{l}\text { NRTL } \\
\end{array}$ & 29.0918 & 1.7525 & 24.5854 & 1.4421 & 34.8301 & 0.9213 & 30.3436 & 0.7611 \\
\hline & UNIQUAC & 217.7131 & 3.9657 & 66.6058 & 0.9113 & 262.5495 & 4.0759 & 77.0007 & 0.9814 \\
\hline & Wilson & 45.5956 & 2.7439 & 25.6068 & 1.3920 & 57.1822 & 1.5622 & 30.9946 & 0.8112 \\
\hline
\end{tabular}

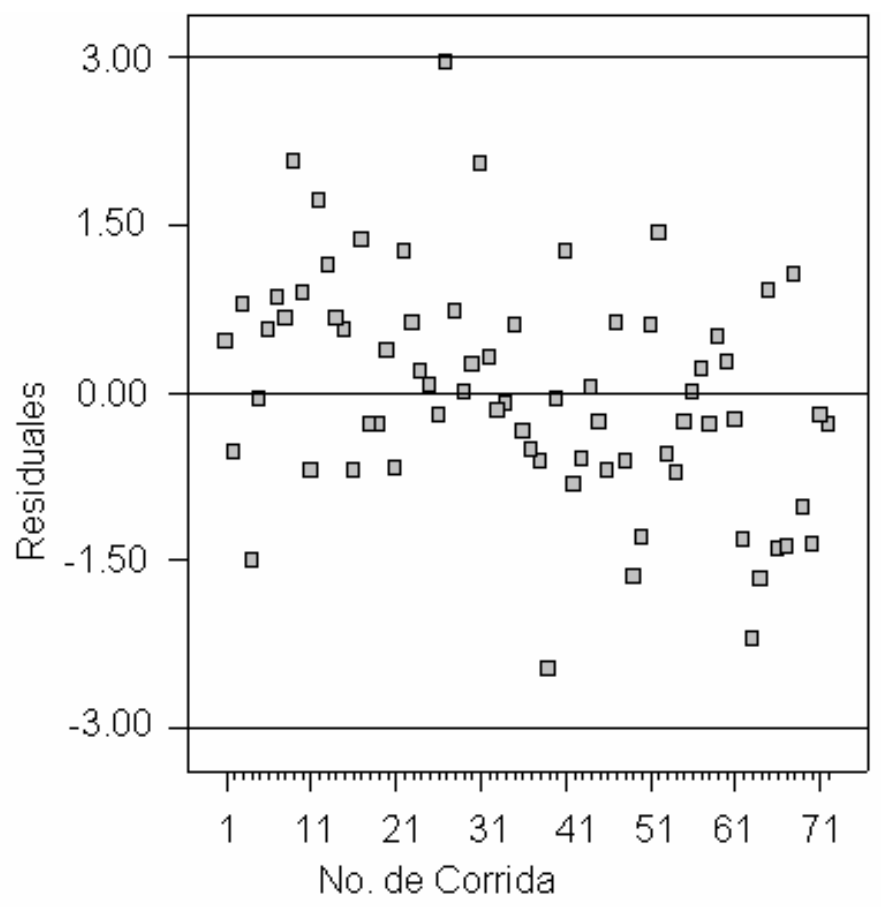

Fig.1. Representación gráfica de los residuales contra las corridas.

Debido a que el tiempo es la variable de interes, es preciso veriicar las posibles respuestas que presenta esta en base a los factores. La Figura 2 muestra el tiempo en función del tipo de modelo (matriz o ciclos), tipo de ecuación para calcular le coeficiente de actividad (NRTL, Wilson, UNIQUAC), variando el tipo de programación y mezcla.

En las Figuras $2 \mathrm{a}$ y $2 \mathrm{~b}$, la mezcla es Acetona cloroformo-Metanol, y se programó las curvas de residuo y Líneas de destilación respectivamente. En las Figuras $2 \mathrm{c}$ y $2 \mathrm{~d}$ la mezcla es Metanol-EtanolBenceno, y se programó las curvas de residuo y Líneas de destilación respectivamente.

El diseño estadístico de experimentos demuestra que, independientemente del tipo de programación, mezcla y modelo de coeficiente de actividad; el modelo de matrices es mucho más rápido que el modelo cíclico. 

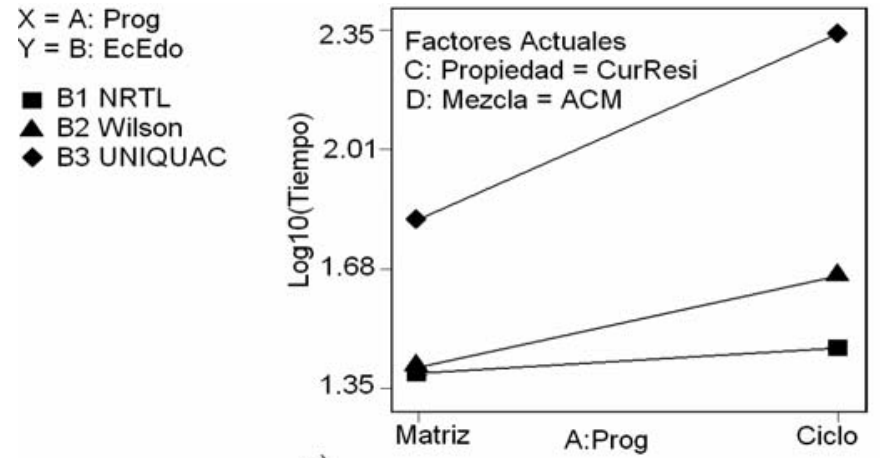

a)

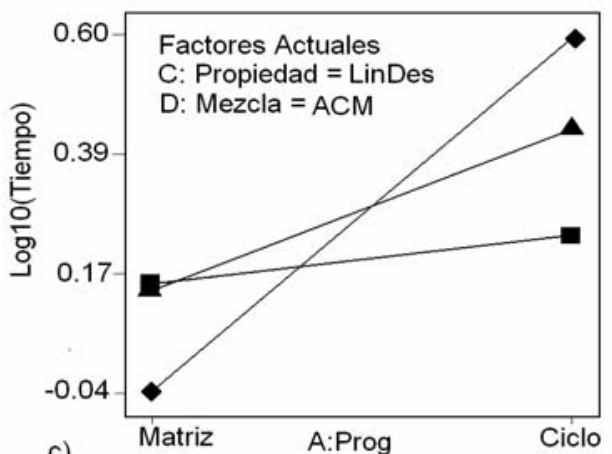

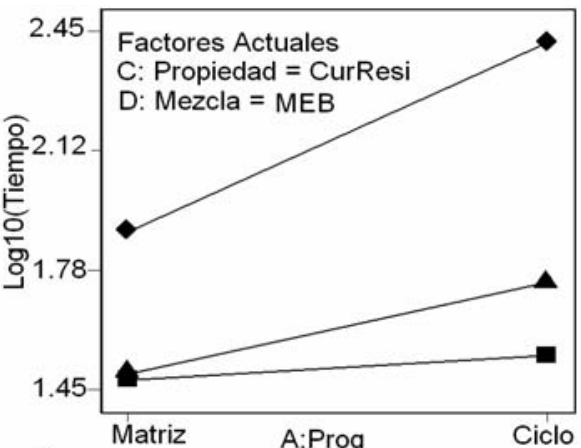

b)

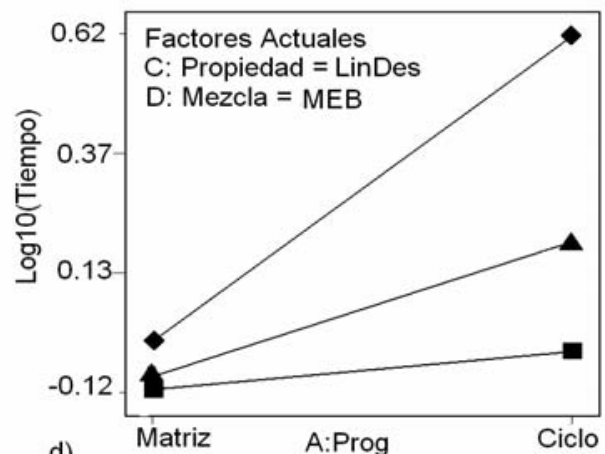

d)

Fig. 2: Representación de la variable de respuesta en función de los factores que la afectan.

\section{CONCLUSIONES}

El análisis estadístico de los experimentos computacionales mostrados aquí, determinó de forma tangible que, la aplicación de modelos matriciales permite obtener resultados con mejor rendimiento y más rápidos, y que a su vez, facilitan y en algunos casos, habilita cálculos mucho más complejos o "pesados". La programación con matrices solo requiere realizar los cálculos una vez para encontrar los resultados, a diferencia de la programación con ciclos, la cual necesita repetir los cálculos dependiendo del número de ciclos anidados y de los componentes, lo que repercute en el incremento del tiempo.

\section{REFERENCIAS}

Abrams, D.S. y J.M. Prausnitz: "Statistical Thermodynamics of Liquid Mixtures: A New Expression for the Excess Gibbs Energy of Partly or Completely Miscible Systems”, AIChE J.: 21(1), 116 (1975).

Doherty, M.F. y M.F. Malone; "Conceptual Design of Distillation Systems", $1^{\text {a }}$ edition, McGraw-Hill chemical engineering series (2001).

Gmehling, J. y U. Onken; "Vapor-Liquid Equilibrium Data Collection", Chemistry Data Series; DECHEMA", Frankfurt, Germany, 1, (1988).

Harding, S.T., C.D. Maranas, C.M. MacDonald y C.A. Floudas; "Locating All Homogeneous Azeotropes in Multicomponent Mixtures", Ind. Eng. Chem. Res.: 36(1), 160-178 (1997).

Kreider, D., R. Kuller y F Perkins; "Introducción al Análisis Lineal”, Fondo Educativo Interamericano, México (1971).

Montgomery, D.C.; "Diseño y Análisis de Experimentos”, 2 ${ }^{\text {da }}$ edición, Limusa Wiley, México, (2004).

Renon, H. y J.M. Prausnitz; "Local Compositions in Thermodynamic Excess Functions for Liquid Mixtures", AlChE J.: 14 (1), 135 (1968).

Widagdo, S. y W.D. Seider; “Azeotropic Distillation”, AIChE J.: 42(1), 96-130 (1996).

Wilson, G.M.; "Vapor-Liquid Equilibrium XI: A New Expression for the Excess Gibbs Energy of Mixing", J. Am. Chem. Soc.: 86, 127, (1964). 